\title{
Comparison of Lagrangian and Eulerian frames of passive scalar turbulent mixing
}

\author{
Paul Götzfried, ${ }^{1}$ Mohammad S. Emran, ${ }^{2}$ Emmanuel Villermaux, ${ }^{3,4}$ and Jörg Schumacher ${ }^{1,5, *}$ \\ ${ }^{1}$ Institut für Thermo- und Fluiddynamik, Postfach 100565, Technische Universität Ilmenau, \\ D-98684, Ilmenau, Germany \\ ${ }^{2}$ Max-Planck-Institut for Dynamik und Selbstorganisation, Am Faßberg 17, D-37077, Göttingen, Germany \\ ${ }^{3}$ Aix Marseille Université, CNRS, Centrale Marseille, IRPHE UMR 7342, 13384 Marseille, France \\ ${ }^{4}$ Institut Universitaire de France, 75231 Paris, France \\ ${ }^{5}$ Tandon School of Engineering, New York University, New York, New York 11201, USA
}

(Received 23 January 2019; published 29 April 2019)

\begin{abstract}
The mixing of a passive scalar in a three-dimensional, statistically stationary turbulent Navier-Stokes flow at a constant and moderate Taylor microscale Reynolds number $R_{\lambda}=$ 42 is studied by means of direct numerical simulations for Schmidt numbers between 1 and 64. The freely decaying passive scalar is represented in two different ways: (1) in the Lagrangian frame of reference as a cloud of up to 4.8 billion individually advected massless tracer particles subject to a stochastic Wiener process along the tracer tracks that describes scalar diffusion or (2) in the standard Eulerian frame of reference as an advection-diffusion equation of the continuum concentration field. In both cases, the scalar is initially seeded in a small cubic subvolume. The mean mixing time $\left\langle t_{s}\right\rangle$ is determined by the mean compressive strain rate $\left\langle\lambda_{3}\right\rangle<0$ which is obtained from the probability density functions of the local finite-time Lyapunov exponents in the Lagrangian frame, $\lambda_{i}(t)$ with $i=1,2$ and 3. The direct comparison of freely decaying Lagrangian and Eulerian passive scalars gives a good agreement of the scalar variance for times $t \lesssim 10\left\langle t_{s}\right\rangle$ and for the probability density functions $P(\Theta, t)$ taken with respect to the whole simulation domain. We also show how the multilayer aggregations of scalar filaments and sheets in the Lagrangian frame are increasingly influenced by the noise due to discreteness with progressing dilution of the initially high tracer particle concentration. This limits the Lagrangian approach in its present form and for the obtainable Schmidt numbers to studies of shorter time periods. A simple one-dimensional advection-diffusion model of a solitary strip is finally applied to the problem at hand to derive the probability density function of the scalar concentration, $P(\Theta, t)$, from the one of the compressive local finite-time Lyapunov exponent, $p\left(\lambda_{3}, t\right)$. Model prediction with and without self-convolution and numerical data of the scalar distributions agree qualitatively, however with quantitative differences particularly for small scalar concentrations. The present Lagrangian approach to passive scalar mixing in turbulence opens the application of more flexible passive scalar injection and boundary conditions and allows to relax the resolution constraints for high-Schmidt number mixing studies.
\end{abstract}

DOI: 10.1103/PhysRevFluids.4.044607

\section{INTRODUCTION}

A better understanding of mixing in turbulent flows is of central interest for numerous applications in engineering and nature. The overall concern in mixing is to understand the interplay between

*joerg.schumacher@tu-ilmenau.de 
the flow kinematics and molecular diffusion, ultimately responsible for reducing the concentration or temperature differences in the flow. If the concentration of the mixed substance $\Theta(\boldsymbol{x}, t)$ is small, then no feedback on the advecting velocity field $\boldsymbol{u}(\boldsymbol{x}, t)$ is expected; the scalar is advected passively in the (turbulent) flow with a kinematic viscosity $v$ and subject to molecular diffusion quantified by the diffusivity constant $D$. The ratio of both material parameters defines the Schmidt number,

$$
\mathrm{Sc}=\frac{v}{D}
$$

Based on the Schmidt number, two different regimes of passive scalar mixing in turbulence are traditionally distinguished. The first regime is the Obukhov-Corrsin regime [1,2] at Sc $\leqslant 1$ for which the passive scalar is advected mostly in the inertial cascade range of the turbulent velocity field, i.e., on scales larger than the viscous Kolmogorov dissipation length, $\ell>\eta_{K}$. The Kolmogorov length $\eta_{K}$ is given by

$$
\eta_{K}=\left(\frac{\nu^{3}}{\langle\epsilon\rangle}\right)^{\frac{1}{4}},
$$

with the mean kinetic energy dissipation rate $\langle\epsilon\rangle$. The kinetic energy dissipation rate field is given by $\epsilon(\boldsymbol{x}, t)=(v / 2)\left(\nabla \boldsymbol{u}+(\nabla \boldsymbol{u})^{T}\right)^{2}$. The second regime is the Batchelor-Kraichnan regime [3,4] for $\mathrm{Sc}>1$ which is of interest in the present work. When Sc is increased beyond unity a growing fraction of scalar filaments is mixed by the spatially smooth fraction of the turbulent flow in the viscous cascade range below the Kolmogorov length $\eta_{K}$. The smallest mean length scale of the passive scalar is then given by the Batchelor scale,

$$
\eta_{B}=\frac{\eta_{K}}{\sqrt{\mathrm{Sc}}}<\eta_{K}
$$

The Batchelor regime is present in the viscous-convective range at scales $\eta_{B}<\ell<\eta_{K}$. The velocity field is spatially smooth in this range and can thus be written as a Taylor expansion, $u_{i}(\boldsymbol{x}, t)=A_{i j}(t) x_{j}$ with the velocity gradient tensor $A_{i j}(t)=\partial u_{i} / \partial x_{j}$ where $i, j=1,2,3$. Here, $\boldsymbol{x}=\left(x_{1}, x_{2}, x_{3}\right)$ or $x_{j}$ in index notation. The motion is mostly a pure straining motion, i.e., local shear layers stretch, twist and fold the scalar filaments. Both regimes of passive scalar turbulence have been studied in many experimental, theoretical and numerical investigations as reviewed in Refs. [5-11].

In case of the Batchelor-Kraichnan regime at high Schmidt numbers, numerical simulations in the Eulerian frame of reference studied the scalar variance spectra, in particular, their decay for wavenumbers $k>\eta_{B}^{-1}[12,13]$, the local isotropy properties of high-Schmidt number mixing [14], the microstructure of the scalar field [15-17], or geometric properties of scalar dissipation sheets [18], to list a few research directions only. Passive scalar mixing was alternatively discussed as an ongoing aggregation process of initially isolated sheets, also denoted as solitary strips or sheets. High-Schmidt-number mixing in this framework has been investigated in turbulent laboratory flows and simulations in simple laminar flows in Refs. [19-24]. In the Lagrangian perspective, the mixing of a passive scalar can be connected to the statistics of the local finite-time Lyapunov exponents (FTLEs), $\lambda_{i}\left(\boldsymbol{x}_{0}, t\right)$ (see also Refs. [6,25-28]), which describes the local stretching and compression rates of material lines along the trajectory of a massless point particle that is seeded in the flow at $\boldsymbol{x}_{0}$ and follows the streamlines of velocity field perfectly.

In this work, we want to describe the passive scalar substance for $\mathrm{Sc} \leqslant 64$ as a very large ensemble of individual particles advected by a three-dimensional (3D) Navier-Stokes flow and subject to Brownian motion. Our study is based on direct numerical simulations (DNS). Such a treatment of a scalar substance has been discussed recently by Okabe et al. [29] in a two-dimensional random sine flow. Here, we will extend this approach to a tracer particle ensemble in a 3D turbulent flow. Our Lagrangian studies are compared with standard Eulerian DNS of passive scalar mixing in which the concentration of the substance is described as a continuum field. This comparison includes the variance and probability density functions (PDFs) of the scalar concentration field 
$\Theta(\boldsymbol{x}, t)$. We also shed light on the advantages and problems of the Lagrangian implementation. One advantage of a discrete particle-based description is a higher flexibility in the implementation of boundary and injection conditions of the scalar, such as absorbing planes or point sources without and with continued (time-dependent) seeding. Limitations of the Lagrangian approach result when a continuum concentration field has to be reconstructed from discrete particle number densities.

The flow domain in the present study is a three-dimensional slab with periodic boundary conditions in all three space directions. Statistically stationary turbulence is sustained by a combination of a random large-scale forcing and a shear forcing which sustains a jetlike turbulent flow in a simple geometry and approximates the passive scalar mixing experiments in a confined jet by Duplat et al. $[20,21,30]$. The flow Reynolds number Re is left constant at a moderate value in our study as listed in Sec. IV of this work.

We will consider two classes of particles advected in the flow. Massless particles that are advected by the velocity field and subject to white-in-time noise along their path are called tracer particles. They describe a passive scalar at finite Sc. Contrary, Lagrangian particles are massless particles that follow the streamlines of the velocity field perfectly without white-in-time noise. The latter class of particles is used in our work for the determination of the local FTLEs and their statistical distribution. The spectrum of the mean FTLE allows us to define a mean Lagrangian mixing time, a typical time scale that will be used for the comparison of the mixing process at different Sc. Also, a simple one-dimensional advection-diffusion model of a solitary strip [20] is finally applied to the problem at hand to derive the PDF of the scalar concentration, $P(\Theta, t)$, from the one of the compressive local FTLE, $p\left(\lambda_{3}, t\right)$. The predicted PDF and self-convolutions of it will also be compared with the numerically obtained scalar distributions. We note here, that the same Lagrangian description of 3D turbulent mixing has been used in Ref. [31] to investigate the particle dispersion in flows at different Sc.

The outline of this work is as follows. In Sec. II, the numerical model is explained in the Eulerian and Lagrangian frames of reference as well as the pseudospectral method for the simulation of the advecting turbulent flow. In Sec. III, the results of the finite-time Lyapunov exponent analysis are presented. In Sec. IV, we compare the Lagrangian and Eulerian model of passive scalar mixing directly for the case of a freely decaying scalar at different Schmidt numbers up to Sc $=64$. Furthermore, we investigate the effect of noise due to discreteness. Section V gives a summary and a brief discussion of future applications of this Lagrangian approach.

\section{NUMERICAL MODEL}

\section{A. Eulerian model of passive scalar}

The equations of motion are solved in a Cartesian slab $L_{x} \times L_{y} \times L_{z}=8 L \times L \times L$ with periodic boundary conditions in all three space directions. Direction $x_{1}$ will be denoted to as the downstream direction. The standard numerical model to study passive scalar turbulence combines an Eulerian description of the velocity field $u_{i}(\boldsymbol{x}, t)$ and the scalar concentration field $\Theta(\boldsymbol{x}, t)$. Therefore, one solves the three-dimensional Navier-Stokes equations for an incompressible fluid together with an advection-diffusion equation for the freely decaying passive scalar (Einstein sum convention is used),

$$
\begin{gathered}
\frac{\partial u_{j}}{\partial x_{j}}=0 \\
\frac{\partial u_{i}}{\partial t}+u_{j} \frac{\partial u_{i}}{\partial x_{j}}=-\frac{1}{\rho_{0}} \frac{\partial p}{\partial x_{i}}+v \frac{\partial^{2} u_{i}}{\partial x_{j}^{2}}+f_{i} \\
\frac{\partial \Theta}{\partial t}+u_{j} \frac{\partial \Theta}{\partial x_{j}}=D \frac{\partial^{2} \Theta}{\partial x_{j}^{2}}
\end{gathered}
$$




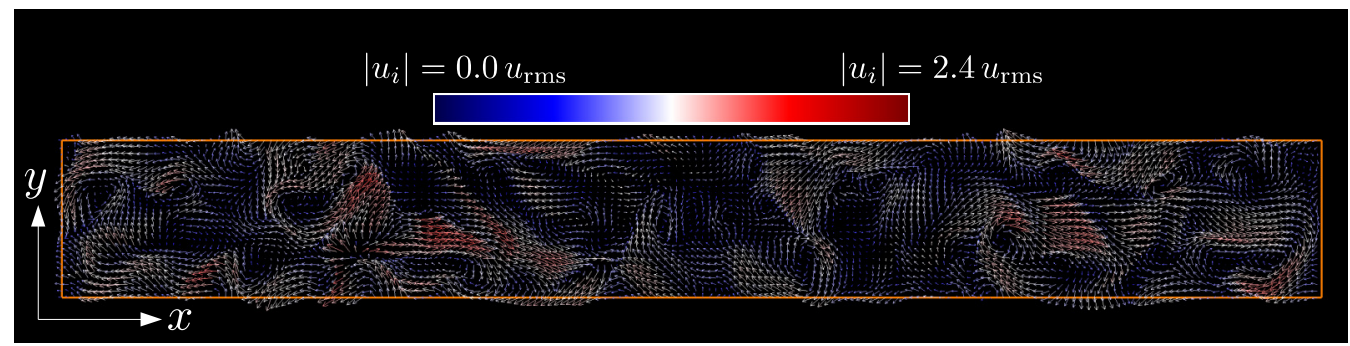

FIG. 1. Snapshot of the advecting turbulent velocity field. Velocity field vectors are projected into the $x-y$ plane at $z=L_{z} / 2$. The arrows are colored by the velocity magnitude which is given in units of the root-mean-square velocity $u_{\text {rms }}$ taken as a combined volume-time average with respect to all three components.

Here, $p$ is the pressure field and $\rho_{0}$ is the constant mass density of the fluid. The passive scalar field is freely decaying. The volume forcing field $f_{i}$ in the Navier-Stokes equations contains here two contributions - a random forcing and the shear forcing-which are applied together and sustain the advecting flow in a statistically stationary state. Figure 1 displays a slice cut of a snapshot of the advecting three-dimensional turbulent flow. The forcing is given by

$$
f_{i}(\boldsymbol{x}, t)=f_{i}^{(L)}(\boldsymbol{x}, t)+f_{i}^{(S)}\left(x_{2}\right) \delta_{i 1} .
$$

This combination of both forcing schemes approximates a confined jet flow in a large tank which was set up in the mixing experiments by Duplat et al. [20,21,30]. The random large-scale forcing $f_{i}^{(L)}$ is implemented in the Fourier space for a subset $K$ of low-wave-number Fourier modes. In accordance with Ref. [32] it is given by

$$
f_{i}^{(L)}(\boldsymbol{k}, t)=\varepsilon_{\text {in }} \frac{u_{i}(\boldsymbol{k}, t)}{\sum_{\boldsymbol{k}_{f}^{(i)} \in K}\left|u_{i}\left(\boldsymbol{k}_{f}^{(i)}, t\right)\right|^{2}} \delta_{\boldsymbol{k}, \boldsymbol{k}_{f}^{(i)}},
$$

with a prescribed injection rate of turbulent kinetic energy $\varepsilon_{\text {in }}$ and the Kronecker $\delta$,

$$
\delta_{\boldsymbol{k}, \boldsymbol{k}_{f}^{(i)}}=1 \quad \text { if } \boldsymbol{k}=\boldsymbol{k}_{f}^{(i)} \quad \text { and } \quad \delta_{\boldsymbol{k}, \boldsymbol{k}_{f}^{(i)}}=0 \text { otherwise. }
$$

The subset $K$ of wave vectors contains the vectors

$$
K=\left\{\boldsymbol{k}_{f}^{(i)}\right\}_{i=1, . ., 48}=\frac{2 \pi}{L}\left\{\left( \pm \frac{1}{\Gamma}, \pm 1, \pm 2\right),\left( \pm \frac{1}{\Gamma}, \pm 2, \pm 2\right)\right\},
$$

which includes all combinations of signs and permutations of components, such as $( \pm 2 / \Gamma, \pm 1, \pm 1)$, $( \pm 2 / \Gamma, \pm 2, \pm 1)$. The downstream wave number is always rescaled by $\Gamma=L_{x} / L_{y}=8$. This random forcing injects turbulent kinetic energy at a fixed rate $\varepsilon_{\text {in }}$ into the flow and was used to sustain the turbulence in isotropic box turbulence. If $f_{i}^{(S)}=0$, then the rate $\varepsilon_{\text {in }}$ prescribes the magnitude of the mean kinetic energy dissipation rate in a statistically stationary regime (see Ref. [32] for detailed discussion). The additional shear forcing $f_{1}^{(S)}$ sustains a cosine shear mode in the flow which is given by

$$
u_{1}^{(S)}\left(x_{2}\right)=-A \cos \left(\frac{2 \pi}{L} x_{2}\right)
$$

and thus results to

$$
f_{1}^{(S)}\left(x_{2}\right)=A v \frac{4 \pi^{2}}{L^{2}} \cos \left(\frac{2 \pi}{L} x_{2}\right)
$$

If $f_{i}^{(L)}=0$ and the Reynolds number sufficiently small, then this forcing sustains a steady and laminar shear flow Eq. (11) in the slab domain [33]. This steady forcing is implemented again 


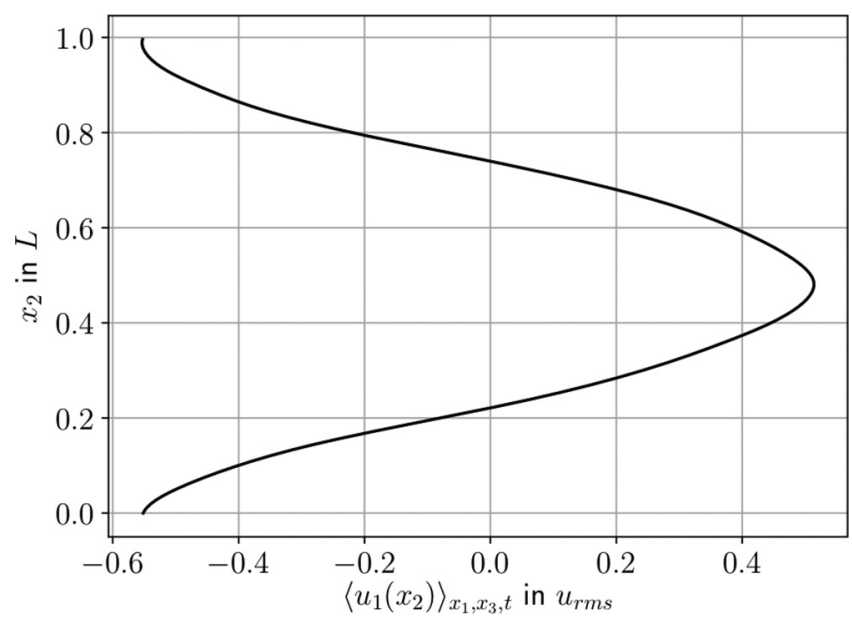

FIG. 2. Mean profile of the streamwise velocity field component $u_{1}$ with respect to $x_{2}$. The profile is obtained by a combined average over coordinates $x_{1}, x_{3}$ and time $t$. This flow property is unchanged for all cases discussed below. Amplitude is given in units of the root-mean-square velocity $u_{\mathrm{rms}}$.

in Fourier space. An amplitude of $A=50$ was chosen throughout the runs. Figure 2 displays the mean profile of the $u_{1}$ velocity component versus $x_{2}$. The figure shows that the additional shear forcing indeed generates a mean jetlike advection as in the laboratory experiments by Duplat et al. $[20,21,30]$. This is established here in a simple domain with periodic boundary conditions and for a uniform computational mesh. The system size, kinematic viscosity and time units are originally set up in cgs units. The results will be discussed in units of box size $L$ and the mean mixing time $\left\langle t_{s}\right\rangle$ that has to be determined first in Sec. III (see also the captions of both tables).

The equations of motion, Eqs. (4)-(6), are solved by an exponentially fast converging pseudospectral scheme. Fields are expanded in Fourier series and the code uses Fast Fourier Transform (FFT) package P3DFFT [34] with a two-dimensional domain decomposition into stencils. The time stepping is done by a second-order integration scheme. The smallest cell size of the uniform grid, $a$, was chosen such that the Batchelor scale $\eta_{B}$ is resolved properly. The spectral resolution satisfies the condition $k_{\max } \eta_{B} \geqslant 1.4$ with $k_{\max }=2 \sqrt{2} \pi N_{2} /(3 L)$ being the maximum wavenumber. We find, that it is a good compromise between sufficient resolution and computational cost.

\section{B. Lagrangian model of passive scalar}

In the Lagrangian description of the passive scalar, we substitute Eq. (6) by a set of $3 \mathcal{N}_{p}$ stochastic ordinary differential equations (also known as Langevin equations), which are given by

$$
\frac{d X_{m}^{l}}{d t}=u_{m}\left(X_{n}^{l}, t\right)+\sqrt{2 D} W_{m}^{l}(t)
$$

Here, $X_{m}^{l}$ is the position of the $l$ th tracer particle and $W_{m}^{l}$ a vectorial, independent stochastic Wiener process with zero expectation value

$$
E\left[W_{m}^{l}(t)\right]=0,
$$

and the following covariance:

$$
\operatorname{Cov}\left[W_{m}^{l}(t), W_{n}^{l}\left(t^{\prime}\right)\right]=\delta_{m n} \min \left(t, t^{\prime}\right) .
$$

Our Brownian motion tests of the weak second-order predictor-corrector scheme [35], which is used to advance the Langevin equations for the tracer particles, are described in the Appendix. The index $l$ runs from 1 to $\mathcal{N}_{p}$. Up to $\mathcal{N}_{p}=4.8 \times 10^{9}$ individual particles were used. In case of a 
diffusion constant $D=0$, a particle would follow the velocity streamlines perfectly and is denoted as a Lagrangian particle. As already stated in the Introduction, we are interested in cases $\mathrm{Sc} \geqslant 1$. Equation (13) describes classical Brownian motion with an additional deterministic drift due to the turbulent velocity field. The advection-diffusion Eq. (6) can be considered as the Fokker-Planck equation for the tracer distribution [36].

A trilinear interpolation is applied to map the Eulerian velocity from the grid to the exact tracer position $X_{m}^{l}$. This operation is required to advect the tracer particles in the turbulent flow. A backward interpolation from the tracer location $X_{m}^{l}$ onto the Eulerian grid is required to construct the concentration field (or a tracer distribution function) $\Theta(x, t)$ by a weighted sum of all nearby particles. In detail, the scalar concentration at the grid position $(i, j, k)$ at a time instant $t_{0}$ is given by

$$
\Theta_{i, j, k}=\frac{\mathcal{N}_{i, j, k} w_{i, j, k}}{N_{1} N_{2} N_{3}}
$$

where $\mathcal{N}_{i, j, k}$ is the number of all tracers in the eight mesh cells $a^{3}$ that have a joint corner in grid point $(i, j, k)$. The corresponding weight factors are determined from the distance of the particle position $X_{m}^{l}$ to the grid point $(i, j, k)$, similar to the velocity in the tri-linear interpolation routine. Thus, the sum over all grid points, which is given by $N_{1} N_{2} N_{3}$, reproduces the total number of tracer particles $\mathcal{N}_{p}$ which are used in the simulation, i.e.,

$$
\sum_{i=1}^{N_{1}} \sum_{j=1}^{N_{2}} \sum_{k=1}^{N_{3}} \mathcal{N}_{i, j, k} w_{i, j, k}=\mathcal{N}_{p}
$$

The discrete particle concentration is smeared out over the scale, $a$ which is of the order of the Batchelor scale, $a \sim \eta_{B}$. The total tracer particle number and thus the mean scalar concentration $\bar{\Theta}$ are conserved quantities in all our simulation runs. The latter is given for the uniform grid at hand by

$$
\bar{\Theta}=\left\langle\mathcal{N}_{i, j, k}\right\rangle=\frac{\mathcal{N}_{p}}{N_{1} N_{2} N_{3}}=\text { const. }
$$

\section{FINITE-TIME LYAPUNOV SPECTRUM}

\section{A. Calculation of the Lyapunov exponents}

The finite-time Lyapunov spectrum comprises all three exponents in a three-dimensional flow. In the following, we describe in brief how they are determined in our work. A small cubic unit volume is assigned to each Lagrangian particle at the beginning which is spanned by three unit vectors $\boldsymbol{e}_{1}$, $\boldsymbol{e}_{2}$, and $\boldsymbol{e}_{3}$ [37]. Once the Lagrangian particle moves with the fluid this attached volume experiences deformations which are determined by the velocity gradient tensor $A_{i k}$ along the tracer track. The computation algorithm starts with a matrix $M_{i j}$ containing the three unit vectors as columns, $\hat{M}=$ $\left[\boldsymbol{e}_{1}, \boldsymbol{e}_{2}, \boldsymbol{e}_{3}\right]$. The evolution equation for $n$ time steps $\Delta t$ of the $l$ th particle with initial $\left(M_{i j}^{0}\right)^{l}=\delta_{i j}$ is given by

$$
\left(M_{i j}^{n}\right)^{l}=\left[\delta_{i k}+\left(\left.\frac{\partial u_{i}}{\partial x_{k}}\right|_{X_{m}^{l}}\right) \Delta t\right]\left(M_{k j}^{n-1}\right)^{l} .
$$

For simplicity, we list the algorithm in Eq. (19) as a simple Euler step method. In the numerical analysis, we take again a predictor-corrector scheme that is second order in time. For each time step a QR-decomposition is done with $\left(M_{i j}^{n}\right)^{l}=\left(Q_{i k}^{n}\right)^{l}\left(R_{k j}^{n}\right)^{l}$, where $\left(Q_{i k}^{n}\right)^{l}$ contains the eigenvectors and $\left(R_{k j}^{n}\right)^{l}$ is an upper triangular matrix for the time step $n \Delta t$. Finally, the finite-time Lyapunov 

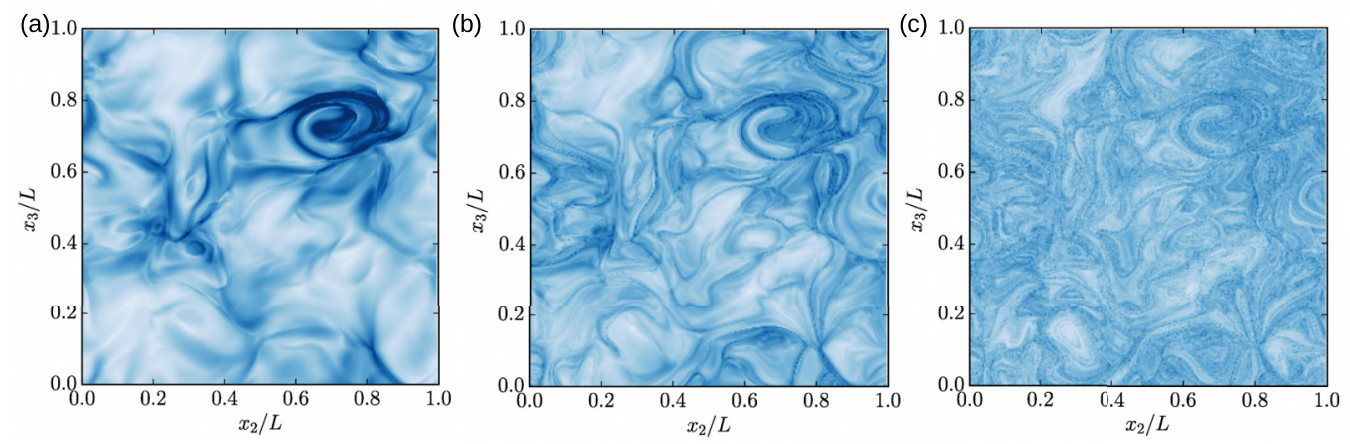

(d)

(e)
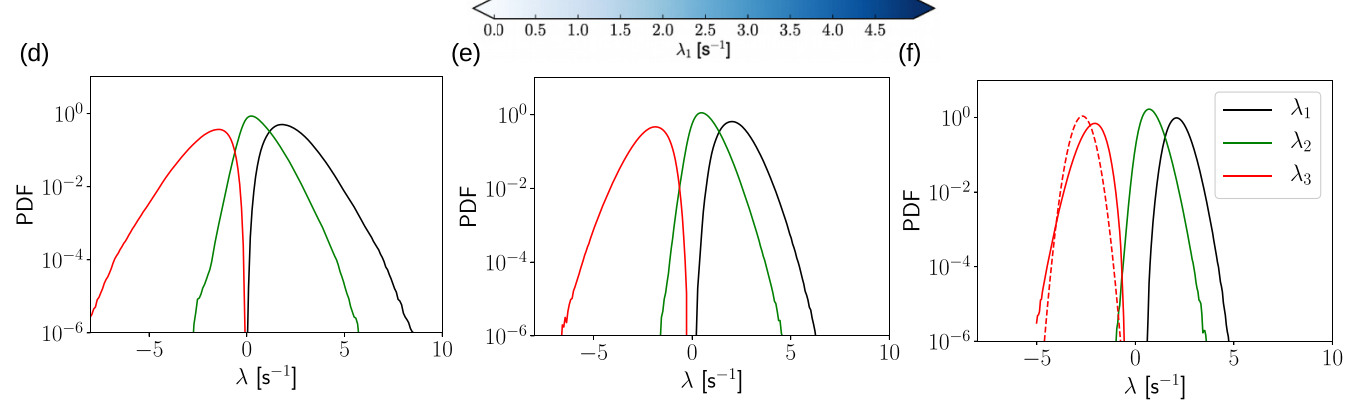

FIG. 3. Finite-time Lyapunov exponents (FTLE) analysis. (a)-(c) Contour plot of the calculated first FTLE, $\lambda_{1}$, for times at $t=0.8, t=2$, and $t=5 \mathrm{~s}$, the dark color represents zones of high stretching rates or where particle trajectories separate most strongly. (d)-(f): The corresponding probability distributions of all three Lyapunov exponents. The first exponent $\lambda_{1}$ is strictly positive and the third exponent $\lambda_{3}$ is strictly negative. With increasing time the distributions converge slowly to the limit of Gaussian distributions with decreasing width. The dashed red line in panel (f) is the corresponding Gaussian distribution. The legend is the same for panels (d)-(f).

exponents are obtained by

$$
\left(\lambda_{i}^{n}\right)^{l}=\frac{1}{n \Delta t} \sum_{m=1}^{n} \ln \left|\left(R_{i i}^{m}\right)^{l}\right| .
$$

Note that there is no Einstein summation rule applied for the index $i$ here. The evaluation of the spectrum has been tested using a $2 \mathrm{D}$ oscillating double gyre flow with two counter rotating vortices [38]. The results are also in agreement with alternative calculation methods [39].

\section{B. Lyapunov spectrum in turbulent flow}

For 3D incompressible turbulence all three FTLE values are non-zero and can be ordered as $\lambda_{1}>\lambda_{2}>\lambda_{3}$. The incompressibility implies also that $\lambda_{1}+\lambda_{2}+\lambda_{3}=0$ (see, e.g., Refs. [40,41]). The largest one is strictly positive, $\lambda_{1}>0$, probing local expansion while the smallest exponent is strictly negative, $\lambda_{3}<0$ probing local compression. The second exponent is positive on average but can have negative and positive values in the course of the dynamical evolution.

We calculate the probability density functions of all three exponents of the Lyapunov spectrum by seeding tracers at the positions of the computational grid initially. A total of $2048 \times 256 \times$ 256 individual Lagrangian particles (which are about 13.4 million) are therefore advanced in time. Figure 3 shows contour plots of the largest finite time Lyapunov exponent $\lambda_{1}$ at different time instants in the top row and the corresponding probability density functions for all three exponents in the bottom row. As visible from the distributions, the largest and smallest exponents are indeed 
strictly positive and negative, respectively. The second exponent is distributed around zero. This indicates that initially spherical blobs will deform to sheetlike structures or filaments which have mostly two stretching and one compression direction until the diffusion becomes dominant. The mean values of the Lyapunov spectrum form a ratio of $\left\langle\lambda_{1}\right\rangle:\left\langle\lambda_{2}\right\rangle:\left\langle\lambda_{3}\right\rangle=4.4: 1:-5.4$. This ratio is consistent with the results in Refs. [40,41]. The relative error of the sum of the mean values is smaller than $2 \times 10^{-4}$ and thus of the order of the chosen numerical integration time step $\Delta t$. The results of the FTLEs will be used, in particular, to predict global mixing statistics of our particular flow. The PDFs of the Lyapunov exponents converge at large times to Gaussian distributions of the form [42]

$$
p\left(\lambda_{3}, t\right)=\sqrt{\frac{t}{4 \pi \kappa}} \exp \left[-\frac{\left(\lambda_{3}-\left\langle\lambda_{3}\right\rangle\right)^{2}}{4 \kappa} t\right],
$$

where $\left\langle\lambda_{3}\right\rangle$ is the most probable exponent of the distribution, and $\kappa$ the variance of the multiplicative process giving rise to it, these two quantities being, in random flows, related to each other by $\left\langle\lambda_{3}\right\rangle=$ $\mathrm{d} \times \kappa$ where $\mathrm{d}$ is the dimension of space (see, e.g., [43]). The corresponding Gaussian is shown in Fig. 3(f). The convergence of the FTLE distributions to Gaussians sharpening in time shows that the uncorrelated stretching histories among the fluid elements become all similar, as suggested by the noisy plot of Fig. 3(c). For completeness, we note that this FTLE spectrum is the same for all runs since the scalars at different $\mathrm{Sc}$ are advected in the same fluid turbulence.

\section{Mean mixing time and local mixing time distribution}

Based on the mean compression rate $\left\langle\lambda_{3}\right\rangle$, we now define a mean mixing time in the Lagrangian frame of reference, $\left\langle t_{s}\right\rangle$, which will be used as the characteristic time unit throughout the rest of this work. The derivation of the time scale follows from a mean compression rate of a scalar filament. We assume that the sheetlike filament obeys a typical initial thickness $s_{0}$. It is compressed at a rate $\gamma=-\left\langle\lambda_{3}\right\rangle$ until it is balanced by molecular diffusion broadening. Equilibrium is achieved at the Batchelor scale $s_{\text {diff, }}$ given by [3]

$$
s_{\mathrm{diff}}=\sqrt{\frac{D}{\gamma}}, \quad \text { where } \quad \gamma=-\left\langle\lambda_{3}\right\rangle .
$$

If one assumes that the compression rate is constant or quasistatic in correspondence with the Batchelor picture [3], then the initial thickness of a scalar sheet decays as $s(t)=s_{0} \exp (-\gamma t)$. The initial filament thickness $s_{0}$ is set here to the extension of our scalar blob which will be discussed in Sec. IV, i.e., $s_{0}=d$. The mean mixing time $\left\langle t_{s}\right\rangle$ is the time beyond which concentration levels in

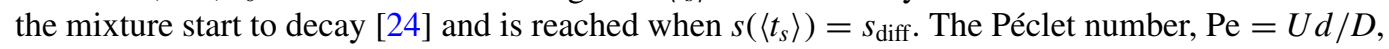
is specified in the present Lagrangian case by

$$
\mathrm{Pe}_{L}=\frac{\gamma d^{2}}{D}
$$

The mean mixing time thus follows with Eq. (22) to

$$
\left\langle t_{s}\right\rangle=\frac{1}{\gamma} \ln \frac{s_{\text {diff }}}{d}=\frac{1}{2 \gamma} \ln \mathrm{Pe}_{L}
$$

and is directly connected to the mean of the third FTLE, which we denote $\gamma$ [26]. This time is an increasing function of Sc grows as is seen in Table I.

Lyapunov exponents are, however, distributed and a distribution of mixing times $P\left(t_{s}\right)$ is associated to their distribution $p\left(\lambda_{3}\right)$. From the distribution of $\lambda_{3}$ and by knowing that $t_{s}\left(\lambda_{3}\right) \approx$ $-\left(2 \lambda_{3}\right)^{-1} \ln \mathrm{Pe}_{L}$ (we discard the weak logarithmic dependence of $t_{s}$ on $\lambda_{3}$ by confusing $-\lambda_{3} d^{2} / D$ with $\mathrm{Pe}_{L}$ ), this distribution is obtained by conservation of probabilities under the following 
TABLE I. List of all simulation runs (Euler $=\mathrm{E}$, Lagrange $=\mathrm{L}$ ). We provide the Schmidt number Sc, the computational grid size and the total number of tracers $\mathcal{N}_{p}$ for Lagrangian cases. The mean mixing time $\left\langle t_{s}\right\rangle$ is given in the last column in seconds. The mean mixing time $\left\langle t_{s}\right\rangle$ is taken as the reference time scale, for both, the Eulerian and Lagrangian runs.

\begin{tabular}{lcccc}
\hline \hline Type & Grid size & Sc & $\mathcal{N}_{p}$ & $\left\langle t_{s}\right\rangle$ \\
\hline E1 & $2048 \times 256^{2}$ & 1 & - & - \\
E2 & $2048 \times 256^{2}$ & 32 & - & - \\
E3 & $4096 \times 512^{2}$ & 64 & - & - \\
L1 & $2048 \times 256^{2}$ & 1 & $1.2 \times 10^{9}$ & 1.00 \\
L2 & $2048 \times 256^{2}$ & 32 & $1.2 \times 10^{9}$ & 1.69 \\
L3 & $4096 \times 512^{2}$ & 64 & $4.8 \times 10^{9}$ & 1.82 \\
\hline \hline
\end{tabular}

substitution rule

$$
P\left(t_{s}\right)=p\left(\lambda_{3}=-\frac{1}{2 t_{s}} \ln \mathrm{Pe}_{L}, t_{s}\right) \times\left|\frac{d \lambda_{3}}{d t_{s}}\right| .
$$

Using Eq. (21) as a fair approximate form of the Lyapunov exponent distribution (which will suit the large $\mathrm{Pe}_{L}$ limit for which the mixing time is large itself), we see that

$$
P\left(x=t_{s} /\left\langle t_{s}\right\rangle\right) \sim \frac{\ln \mathrm{Pe}_{L}}{x^{3 / 2}} \exp \left[-\frac{\ln \mathrm{Pe}_{L}}{8} x\left(\frac{1}{x}-1\right)^{2}\right],
$$

where use has been made of the fact that $\left\langle\lambda_{3}\right\rangle / \kappa$ is a constant. This distribution presents an exponential tail at large excursion $P(x \gg 1) \sim \exp \left[-\frac{\ln \mathrm{Pe}_{L}}{8} x\right]$ (see Refs. [20,27]), and a rapid fall-off for small mixing times $P(x \ll 1) \sim \exp \left[-\frac{\ln \mathrm{Pe}_{L}}{8 x}\right]$, reminiscent of the rare but strong events leading to fast mixing. Obviously, since the mixing time sets the time above which concentrations decay, the distribution of $t_{s}$ is associated with a distribution of concentration at a given instant of time [43], which we will analyze below.

\section{SIMULATION RESULTS}

\section{A. Setup of Eulerian and Lagrangian runs}

We have conducted a series of six DNS which are grouped in three Lagrangian (L) and three Eulerian (E) runs of freely decaying passive scalar turbulence. The advecting turbulent velocity field is the same in all simulations and kept in a statistically stationary state by a combination of both forcing schemes that have been introduced in Sec. II A. Schmidt numbers take values of 1, 32, and 64. All important simulation parameters are listed in Tables I and II.

In the Eulerian case, the scalar concentration inside the blob of side length $d$ is one and zero elsewhere. A sufficiently smooth crossover is established by

$$
\Theta_{s}\left(x_{i}\right)=\frac{1}{\exp \left[\sigma\left(x_{i, s}-x_{i}\right)\right]+1} \quad \text { and } \quad \Theta_{e}\left(x_{i}\right)=\frac{1}{\exp \left[\sigma\left(x_{i}-x_{i, e}\right)\right]+1},
$$

where $x_{i, s}$ and $x_{i, e}=x_{i, s}+d$ are the coordinates of the blob volume similar to the seeding intervals for Lagrangian tracers. The initial scalar concentration follows by

$$
\Theta\left(\boldsymbol{x}, t_{0}\right)=\prod_{i=1}^{3} \Theta_{s}\left(x_{i}\right) \Theta_{e}\left(x_{i}\right) .
$$

The coordinates are $x_{1, s}=0.875 L$ and $x_{2, s}=x_{3, s}=0.375 L$. Parameter $\sigma=10$ is used in Eq. (27) to reduce errors due to the Gibbs phenomenon [44]. Figure 4 shows the initial setup and snapshots of the subsequent mixing process under the influence of statistically stationary fluid turbulence with 
TABLE II. List of important constants, reference values and initial simulation parameters. The Reynolds number is defined as $\operatorname{Re}=u_{\mathrm{rms}} L / v$. The Taylor microscale Reynolds number is given by $R_{\lambda}=$ $\sqrt{5 /(3\langle\epsilon\rangle v)} u_{\mathrm{rms}}^{2}$. The specific domain length is in our setup $L=12.8 \mathrm{~cm}$. The kinematic viscosity is that of air. The root mean square velocity $u_{\mathrm{rms}}=6.64 \mathrm{~cm} \mathrm{~s}^{-1}$.

\begin{tabular}{lccc}
\hline \hline Quantity & Symbol & Reference Unit & Value \\
\hline Domain length in $x_{1}$ & $L_{1}$ & $L$ & 8 \\
Domain length in $x_{2}, x_{3}$ & $L_{2}, L_{3}$ & $L$ & 1 \\
Kolmogorov scale & $\eta_{K}$ & $L$ & $1.04 \times 10^{-2}$ \\
Batchelor scale for Sc $=32$ & $\eta_{B}$ & $L$ & $1.84 \times 10^{-3}$ \\
Batchelor scale for Sc $=64$ & $\eta_{B}$ & $L$ & $1.25 \times 10^{-3}$ \\
Taylor microscale & $\lambda$ & $L$ & 0.13 \\
Integral length scale & $l$ & $L$ & 0.75 \\
Reynolds number & $\mathrm{Re}$ & & 581 \\
Taylor microscale Reynolds number & $R_{\lambda}$ & $L$ & 42 \\
Grid size & $a$ & $u_{\mathrm{rms}}^{3} / L$ & $3.91 \times 10^{-3}$ \\
Energy injection rate & $\epsilon_{\mathrm{in}}$ & $u_{\mathrm{rms}}^{3} / L$ & 0.22 \\
Mean energy dissipation rate & $\langle\epsilon$ & $L$ & 0.473 \\
Side length of the blob & $d$ & 0.25 \\
\hline \hline
\end{tabular}

additional shear forcing. The blob is initially localized and then dispersed by turbulence mostly into sheetlike structures that propagate downstream. Therefore, it is stretched locally in two directions and compressed in a third one. This initial configuration is the same for all runs.

\section{B. Comparison of variance for freely decaying scalar}

In this subsection, we compare the Eulerian and the Lagrangian description of the freely decaying passive scalar. The first statistical measure that is applied here for comparison is the variance of the
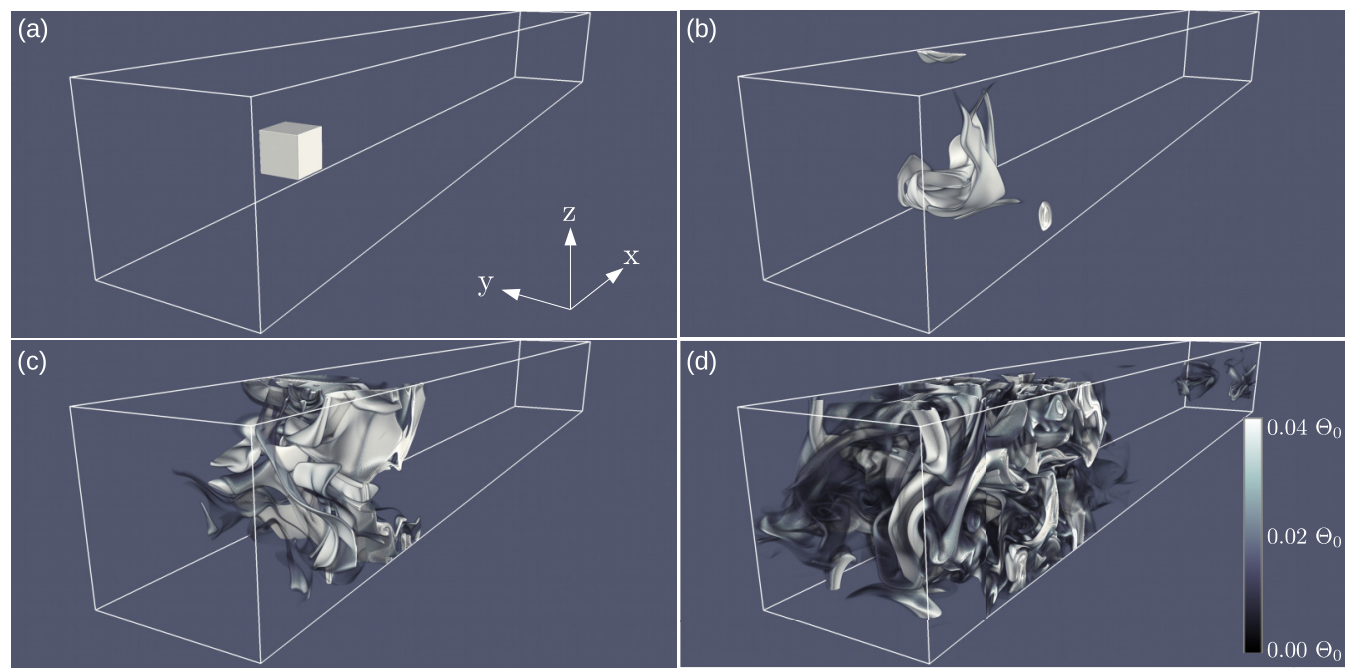

FIG. 4. Mixing of a passive scalar in a periodic slab domain. The passive scalar is simulated in the Lagrangian frame of reference as an ensemble of 4.8 billion individual tracers with additional noise (Langevin equation). Initially all tracers start in a small subdomain. We display the configuration for times $t=0\left\langle t_{s}\right\rangle$, $0.55\left\langle t_{s}\right\rangle, 1.10\left\langle t_{s}\right\rangle$, and $2.20\left\langle t_{s}\right\rangle$. The Schmidt number is $\mathrm{Sc}=64$. The additional shear forcing causes a mean advection in $x_{1}$ direction. 

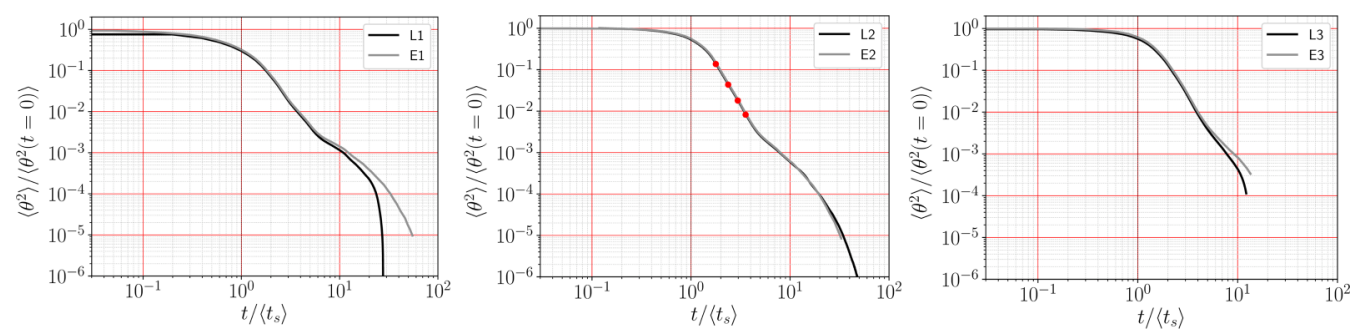

FIG. 5. Variance of scalar concentration fluctuations, $\theta$, versus time. Time is given in units of the mean mixing time $\left\langle t_{s}\right\rangle$. The plot shows the direct comparison of Eulerian and Lagrangian runs for freely decaying scalar turbulence at $\mathrm{Sc}=1$ (left), $\mathrm{Sc}=32$ (middle), and $\mathrm{Sc}=64$ (right). For $t /\left\langle t_{s}\right\rangle \gtrsim 5$ an overlap of the scalar filaments occurs where they re-enter the simulation domain in the downstream direction $x_{1}$, due to the applied periodic boundary conditions. This overlapping or aggregation causes the change in the decay rate. The four highlighted data points in the middle panel indicate time instants for an analysis which will be discussed in Sec. IV D.

scalar concentration fluctuations, $\theta=\Theta-\bar{\Theta}$, which is given by

$$
\left\langle\theta^{2}(t)\right\rangle=\left\langle\Theta^{2}(t)\right\rangle-\bar{\Theta}^{2},
$$

and where $\langle\cdot\rangle$ stands for a volume average over the whole simulation domain. For the present setups, $\bar{\Theta}$ is constant in time. We recall that the time evolution of the mean square of $\theta$ is given by the following balance equation:

$$
\frac{1}{2} \frac{\partial\left\langle\theta(t)^{2}\right\rangle}{\partial t}=-D\left\langle\left(\frac{\partial \theta}{\partial x_{j}}\right)^{2}\right\rangle_{V}=-\varepsilon_{D}(t),
$$

In the limit $t \rightarrow \infty$ this variance value will approach zero. On the right-hand side of the equation, the mean dissipation rate $\varepsilon_{D}(t)>0$ of the scalar fluctuation field $\theta$ is given. All boundary flux terms sum up to zero for periodic boundary conditions. The scalar dissipation rate relies heavily on how well the scalar field is stirred, i.e., where the scalar isosurfaces are stretched and folded strongly by the turbulent flow until the aggregated sheets with large concentration gradients start to become subject to molecular diffusion.

Figure 5 shows the comparison of the time behavior of the variance of the scalar fluctuations, $\left\langle\theta^{2}(t)\right\rangle$, for Schmidt numbers of $\mathrm{Sc}=1,32$, and 64 normalized by the initial value. The three double-logarithmic plots contain the Eulerian and Lagrangian representation of the passive scalar. The time is normalized by the corresponding mean mixing time scale $\left\langle t_{s}\right\rangle$ in each panel (see Eq. (24) and Table I). Overall, a good agreement is found when comparing the curves of the Eulerian and Lagrangian models up to about $10\left\langle t_{s}\right\rangle$ for all three pairs. For a dispersing mixture in its initial phase, the scalar variance is proportional to the square of the mean and thus also to the maximum concentration. This has been shown by Duplat et al. [21]. The experimental data in Refs. [21] and [24] agree with the time behavior displayed in Fig. 5.

A change in the slope of all curves after a few mixing times can be observed in the figure. This change is caused by the periodic boundary conditions and a resulting additional enhanced aggregation of scalar sheets across the faces in downstream direction $x_{1}$. It is observable for $t \approx 6\left\langle t_{s}\right\rangle$ at $\mathrm{Sc}=1$, for $t \approx 5\left\langle t_{s}\right\rangle$ at $\mathrm{Sc}=32$, and for $t \approx 5\left\langle t_{s}\right\rangle$ at $\mathrm{Sc}=64$. Our finding is due to the following points: first, the advecting flow is the same in all cases, secondly, the mixing time $\left\langle t_{s}\right\rangle$ increases with $\mathrm{Sc}$, and thirdly, the convolution of scalar sheets is strongest for the highest Sc. Thus, the change of the slope is weakest for $\mathrm{Sc}=64$ and sets in at the largest convective time. With progressing time, the curves of the Lagrangian and Eulerian runs start to deviate of each other.

For the Lagrangian case an offset was subtracted which is related to the discrete character of the tracer field for the reconstruction of the scalar concentration. This circumstance becomes ever 
more important since the scalar is increasingly diluted as the time progresses. Such limitations due to the discreteness were already found and investigated in Okabe et al. [29]. To conduct a statistical analysis, one has to subtract this offset. It occurs, on the one hand, due to a finite number of particles and, on the other hand, due to the finite grid size at which the concentration field $\theta$ is evaluated. Okabe et al. gave their tracer particles a finite lifetime which is related to times when mixing starts to be dominated by diffusion. The "aged" particles are then excluded from the analysis.

The error due to discreteness can be estimated and is dependent on the average concentration, as already defined in Eq. (18). For example, for cases L1 and L2 with 1.2 billion tracer particles and the chosen computational mesh of $2048 \times 256 \times 256 \approx 1.34 \times 10^{8}$ points one gets a constant value of $\bar{\Theta} \approx 8.9$, for case L3 with a mesh of $4096 \times 512 \times 512 \approx 10^{9}$ and 4.8 billion tracers a value of $\bar{\Theta} \approx 4.5$. For the Eulerian case, one gets

$$
\lim _{t \rightarrow \infty}\left\langle\theta^{2}(t)\right\rangle=0 \quad \text { or } \quad \lim _{t \rightarrow \infty}\left\langle\Theta^{2}(t)\right\rangle=\bar{\Theta}^{2} .
$$

For the Lagrangian case with a discrete particle field, the well-mixed final state still shows fluctuations around $\Theta=\bar{\Theta}$, since the turbulent flow still advects particles across the computational mesh cell with $V_{\text {mesh }}=a^{3}$. This is a manifestation of the noise due to the discrete number of particles.

\section{Comparison of scalar distributions for Eulerian and Lagrangian freely decaying cases}

Which scalar PDF $P(\Theta)$ can be expected for times very large with respect to the mean mixing time? The final tracer distribution will be a binomial distribution which is given by

$$
p(k)=\left(\begin{array}{c}
\mathcal{N}_{p} \\
k
\end{array}\right) p^{k}(1-p)^{\mathcal{N}_{p}-k},
$$

where $p(k)$ is the probability to find $k$ tracer particles in the considered grid cell $a^{3}$, and $p=$ $1 /\left(N_{1} N_{2} N_{3}\right)$ is the probability to find the selected particle in a particular grid cell. The binomial distribution can be approximated by a Poisson distribution for small $p$ and a large number of tracer particles $\mathcal{N}_{p} \gg 1$. Since this condition is valid here, the probability is defined as

$$
p(k)=\frac{\bar{\Theta}^{k}}{k !} e^{-\bar{\Theta}} .
$$

For the limit of a large mean number of particles $\bar{\Theta} \gg 1$, this distribution will eventually converge to a $\delta$ distribution $\delta(k-\bar{\Theta})$. The background fluctuation due to noise is then the standard deviation of the Poisson distribution and follows from the numbers of the last subsection for cases L1 and L2 (L3) to $\sigma=\sqrt{\bar{\Theta}} \approx 2.99(2.11)$. In the case of total homogenization each grid box would thus contain on average $\bar{\Theta} \pm \sigma \approx 9 \pm 3(4 \pm 2)$ tracer particles.

Which scalar PDF $P(\Theta)$ follows for the initial phase of the mixing process? Both cases $(\mathrm{E}$ and L) are shown in Fig. 6, where concentration distributions are plotted for different time instants given in units of the corresponding mean mixing time $\left\langle t_{s}\right\rangle$. Initially, there is a pronounced peak at the seeding concentration level for both cases. This nearly bimodal distribution is expected and has been also seen in laboratory experiments [20]. The concentration is normalized by the initial

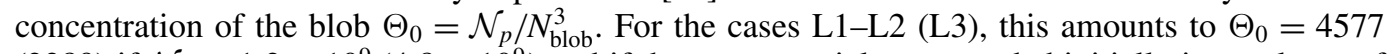
(2289) if $\mathcal{N}_{p}=1.2 \times 10^{9}\left(4.8 \times 10^{9}\right)$ and if the tracer particles are seeded initially in a volume of $\mathcal{N}_{\text {blob }}^{3}=64^{3}\left(128^{3}\right)$ mesh cells. A slight difference can be detected at the highest concentration level $\Theta / \Theta_{0} \approx 1$. The local maximum is less spike-shaped for the Lagrangian cases in comparison to the Eulerian ones. The absence of the initial spike at the highest concentration level for L1 to L3 is a combined effect of limited number of particles and the discreteness error.

After times $t>\left\langle t_{s}\right\rangle$ the peak of the PDF vanishes and a distribution with an exponential tail develops. It is seen that the PDFs agree well for these instants and for all Schmidt numbers. Furthermore, values slightly larger than the blob concentration $\Theta_{0}$ are found for the Lagrangian 
(L1)

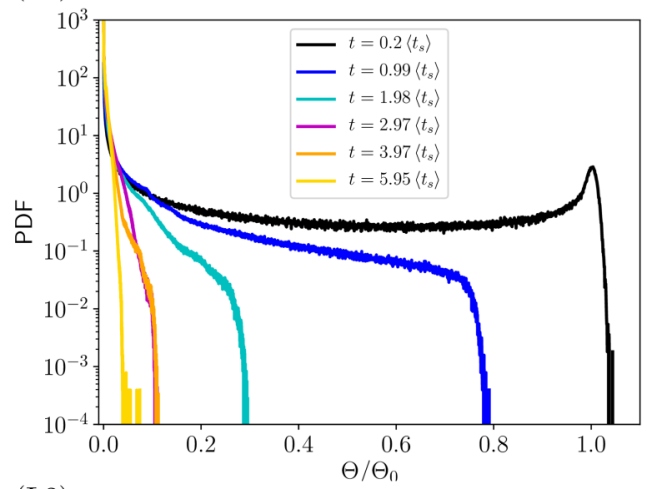

(L2)

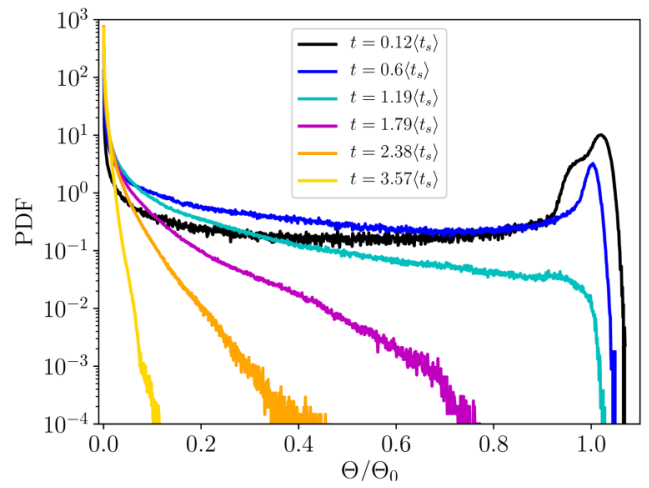

(L3)

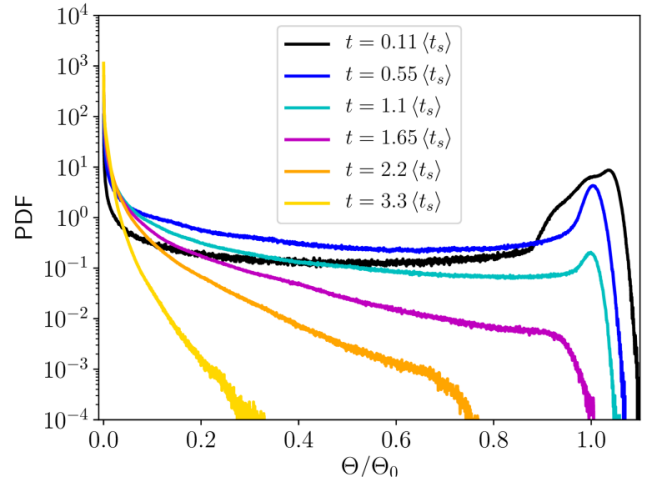

(E1)

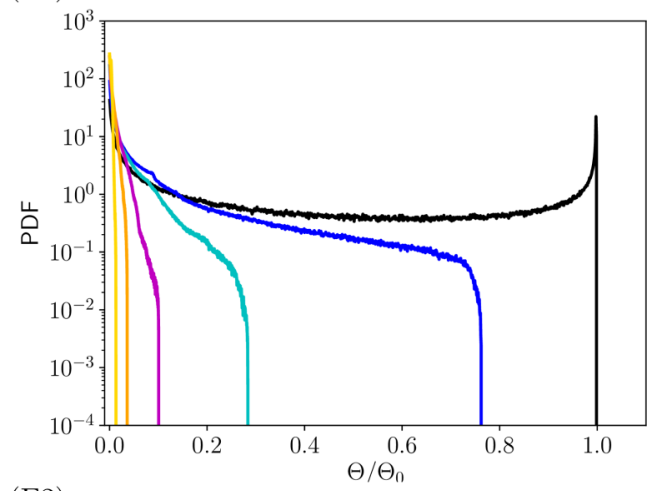

(E2)

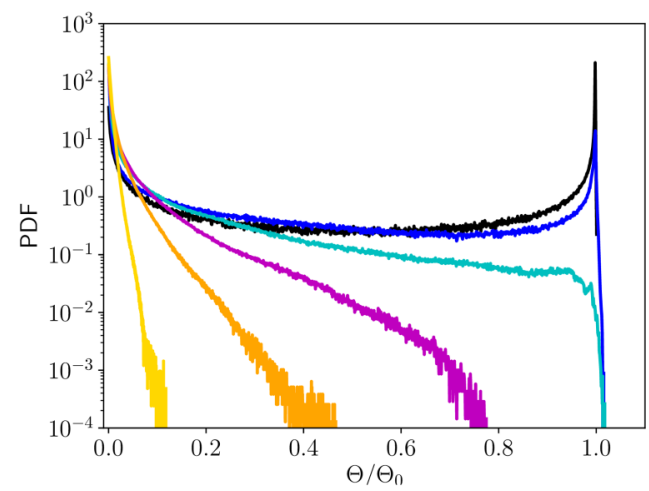

(E3)

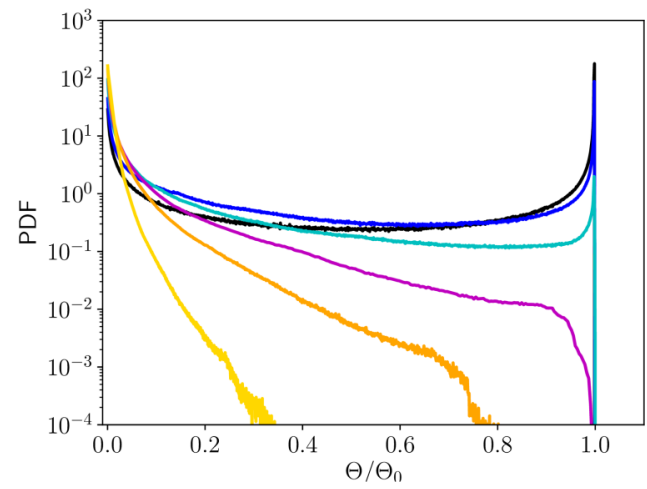

FIG. 6. Probability density functions (PDFs) of the passive scalar concentration taken at different times of the evolution for different Schmidt numbers. The left column shows the Lagrangian cases L1, L2, L3, the right column the Eulerian cases E1, E2, E3. Top row: $\mathrm{Sc}=1$. Middle row: $\mathrm{Sc}=32$. Bottom row: $\mathrm{Sc}=64$. At the beginning, the scalar PDF is a bimodal distribution with entries at $\Theta / \Theta_{0}=0$ and 1 only.

case in the early stage which can be related again to the noise due to discreteness and thus there is a probability to find more particles in a chosen bin.

A visual comparison of cases E3 and L3 is done in Fig. 7 where the scalar concentration is plotted for a vertical cut through the slab sufficiently far away from the seeding region at $x=4 d$. The early stage (top row) of the evolution gives a nearly perfect agreement between L3 (left) and E3 (right). At the later stage (see bottom row), the noise due to discreteness starts to affect the contours of L3. 

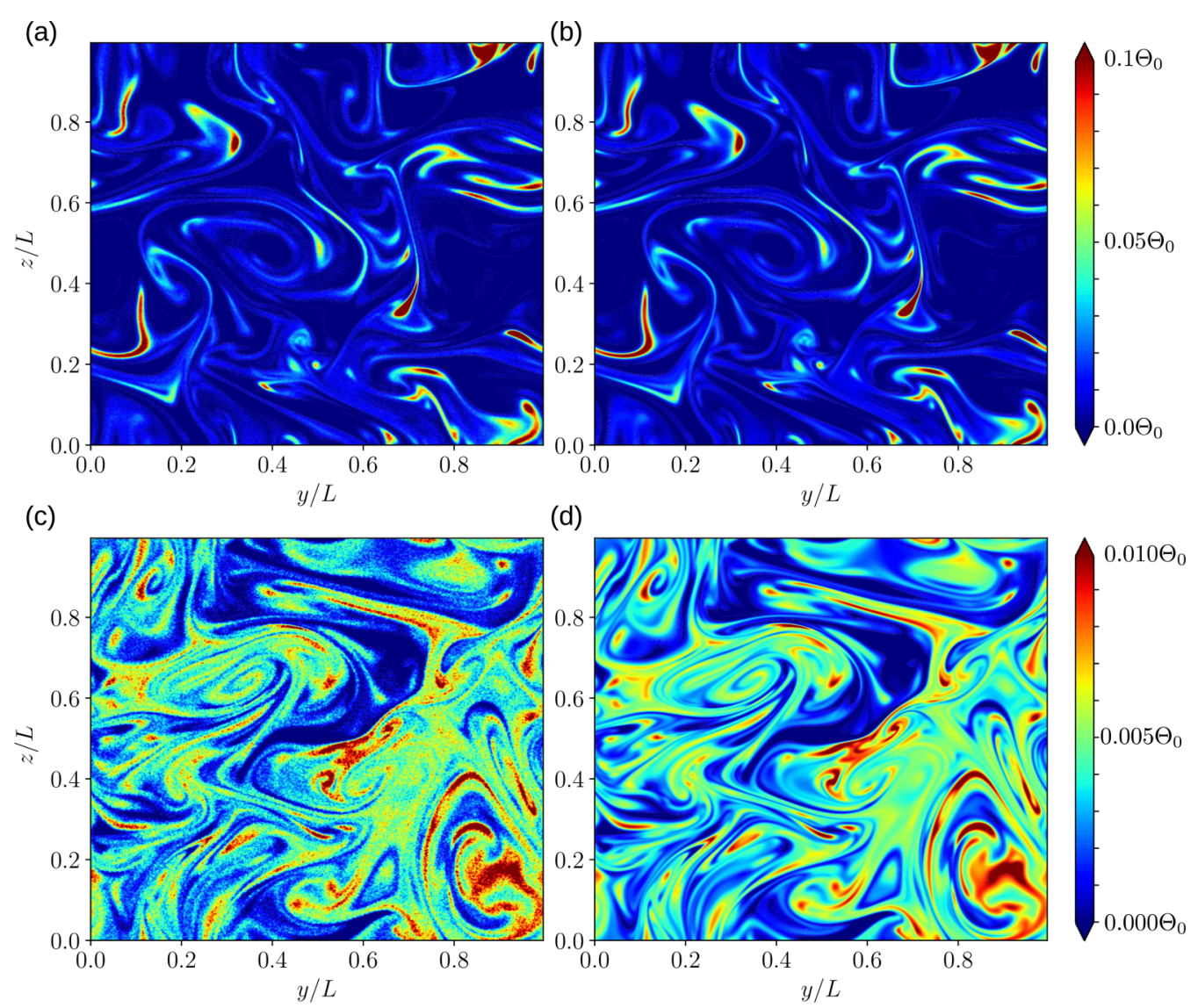

FIG. 7. Isocontour plots of the concentration field for the cases L3 and E3 of a decaying passive scalar with Schmidt number Sc $=64$ at $x=4 d$ with $d / L=0.25$. The field is represented by 4.8 billion individual tracer particles (left column) or by an Eulerian scalar field advected by the fluid flow (right column) for times $t=2.75\left\langle t_{s}\right\rangle$ (top row) and $t=5.5\left\langle t_{s}\right\rangle$ (bottom row).

However, the majority of features of the contour plot, such as larger low-concentration voids, are still well comparable. It would require an adaptive and continued seeding of new tracer particles to compensate for the ongoing dilution by the advection process as done in Martínez-Ruiz et al. [23] for a simple laminar flow.

\section{Derivation of scalar distribution from Lyapunov exponent distribution}

Finally, we want to reconstruct the scalar concentration distribution $P(\Theta, t)$ from the distribution of the Lagrangian compression rates, $p\left(\lambda_{3}, t\right)$ and the evolution of the maximal concentration, $\Theta_{\max }$. We rely here on an approach by Ranz [45] that introduces new coordinates-two along the scalar sheet (or solitary strip), $\xi_{1}$ and $\xi_{2}$, and one perpendicular to the sheet, $\zeta$. This coordinate system is aligned with the local eigenframe of the rate of strain tensor. The sheet will be stretched and compressed locally as determined by the local Lyapunov exponents $\lambda_{1}, \lambda_{2}$, and $\lambda_{3}$. Since diffusion is important in the most compressive direction only and can be neglected in the other two directions, the passive scalar advection-diffusion Eq. (6) simplifies to a one-dimensional equation, which is given by

$$
\frac{\partial \Theta}{\partial t}+\gamma \zeta \frac{\partial \Theta}{\partial \zeta}=D \frac{\partial^{2} \Theta}{\partial \zeta^{2}}
$$




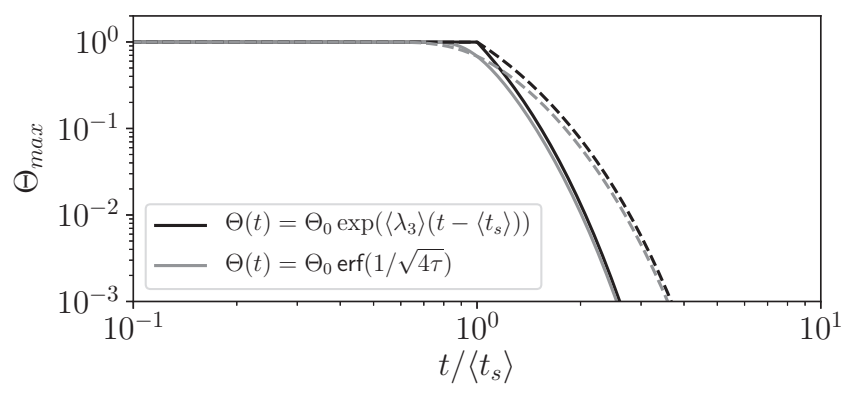

FIG. 8. Maximum scalar concentration versus time. Comparison of the approximation (gray curves) and the error function (black curves) for the cases $\mathrm{Sc}=1$ (dashed lines) and $\mathrm{Sc}=32$ (solid lines).

when written along the most probable stretching trajectory for which $\left\langle\lambda_{3}\right\rangle=-\gamma$. This equation now contains only derivatives perpendicular to the sheet surface and has been in detail discussed in Refs. $[19,20,22]$. The compressive strain $\gamma$ is such that

$$
\gamma=-\frac{d \ln s(t)}{d t}
$$

Equation (34) can be solved analytically $[19,20,22,45]$ once the following transformations of time and space coordinates are introduced. One defines a so-called warped time $\tau$ and a normalized perpendicular coordinate $\tilde{\zeta}$, both given by

$$
\tau=D \int_{0}^{t} \frac{1}{s\left(t^{\prime}\right)^{2}} d t^{\prime}=\underbrace{\frac{D}{2 \gamma d^{2}}}_{\left(2 \mathrm{Pe}_{L}\right)^{-1}}[\exp (2 \gamma t)-1], \quad \text { with } \quad \tilde{\zeta}=\frac{\zeta}{s(t)} .
$$

These substitutions simplify Eq. (34) to a standard heat equation in the new coordinates

$$
\frac{\partial \Theta}{\partial \tau}=\frac{\partial^{2} \Theta}{\partial \tilde{\zeta}^{2}} .
$$

Starting from a strip of width $d$ and uniform concentration $\Theta_{0}$, Eq. (37) has a solution which is given by

$$
\Theta(\tilde{\zeta}, t)=\frac{\Theta_{0}}{2}\left[\operatorname{erf}\left(\frac{\tilde{\zeta}+1 / 2}{\sqrt{4 \tau}}\right)-\operatorname{erf}\left(\frac{\tilde{\zeta}-1 / 2}{\sqrt{4 \tau}}\right)\right] .
$$

The maximum concentration at the center of the strip (i.e., in $\zeta=0$ ) is given by

$$
\Theta_{\max }=\Theta_{0} \operatorname{erf}\left(\frac{1}{\sqrt{4 \tau}}\right),
$$

and thus $\Theta_{\max } \approx \Theta_{0}$ for times $t<t_{s}$ while for $t>t_{s}$, it follows approximately that

$$
\Theta_{\max } \approx \Theta_{0} \exp \left[-\gamma\left(t-\left\langle t_{s}\right\rangle\right)\right] .
$$

Both functions are shown in Fig. 8 and almost coincide for $t>\left\langle t_{s}\right\rangle$. The solid and dashed lines represent cases where $\mathrm{Sc}=32$ and 1, respectively. The temporal decay agrees also with scalar mixing experiments as seen, e.g., in Fig. 5 of Ref. [24]. Similar to the scalar variance, the decay of $\Theta_{\max }$ starts for $t \gtrsim\left\langle t_{s}\right\rangle$.

Under the two assumptions that (i) concentrations are assimilated to the maximal strip concentration $\Theta_{\max }$ in Eq. (40) written for any compression rate $\lambda_{3}$ as

$$
\Theta \equiv \Theta_{\max } \approx \Theta_{0} \exp \left[\lambda_{3}\left(t-\left\langle t_{s}\right\rangle\right)\right],
$$



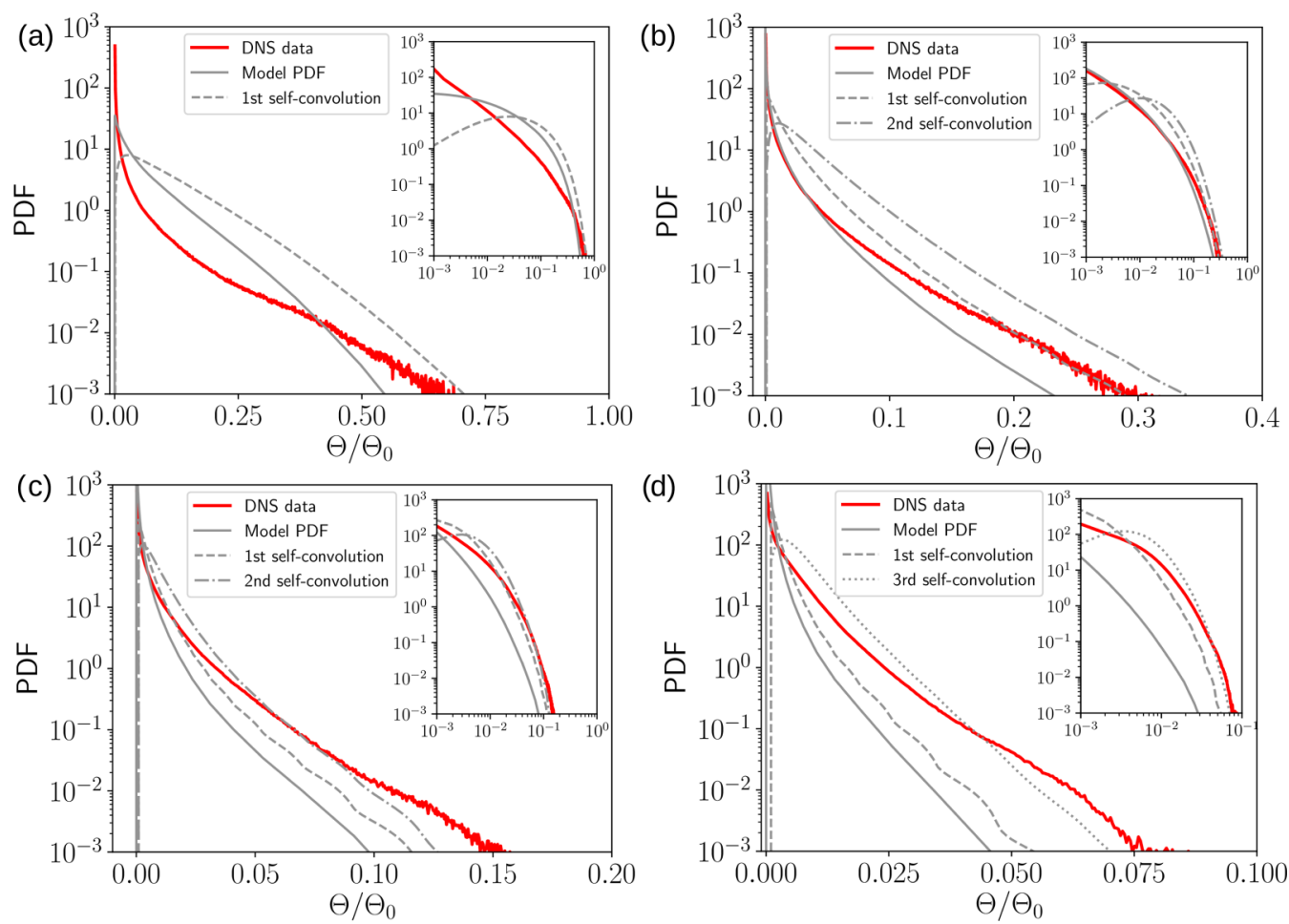

FIG. 9. Scalar distribution (red lines) shown together with the distribution based on the prediction from the smallest Lyapunov exponent (solid gray lines) as defined in Eq. (43). The additional gray lines represent (multiple) self-convolution operations of the passive scalar PDFs. Times are $t=1.79\left\langle t_{s}\right\rangle$ in (a), $2.38\left\langle t_{s}\right\rangle$ in (b), $2.98\left\langle t_{s}\right\rangle$ in (c), and $3.57\left\langle t_{s}\right\rangle$ in (d). The insets in the figure replot the corresponding data on logarithmic axes.

and (ii) that the mixing times are all assimilated to $\left\langle t_{s}\right\rangle$ although $\lambda_{3}$ is distributed, the scalar concentration PDF $p(\Theta, t)$ can now inferred using again probability conservation as

$$
p(\Theta, t) d \Theta=p\left(\lambda_{3}, t\right) d \lambda_{3},
$$

providing

$$
p(\Theta, t)=\frac{1}{\left(t-\left\langle t_{s}\right\rangle\right) \Theta} p\left[\lambda_{3}=\frac{1}{t-\left\langle t_{s}\right\rangle} \ln \left(\Theta / \Theta_{0}\right), t\right] .
$$

These resulting PDFs are now compared to the scalar distributions of the L2 case in Fig. 9. Comparison times are chosen such that the sheets are still not affected by strong overlapping via the periodic faces in the downstream direction $x_{1}$. These times are also marked as symbols in the decay curve in the middle panel of Fig. 5. It can be seen that the model prediction underestimates the tails in all cases, which are an indicator of unmixed higher-concentration fragments with maximal concentration $\Theta_{\max }$. One reason for the deviations is the fact that scalar concentration filaments can overlap. Such an overlap of filaments (also denoted as self-convolution) becomes increasingly relevant as the time progresses. Self-convolution was discussed in Ref. [43] for the mixing of a solitary strip in two dimensions in case of very large Péclet number. Here, we want to apply this concept to a three-dimensional scalar blob. The self-convolution occurs randomly $[19,30]$ and is given by

$$
p^{\otimes 2}(\Theta, t)=\int_{\Theta^{\prime}=0}^{\Theta^{\prime}=\Theta_{0}^{\prime}} p\left(\Theta^{\prime}\right) p\left(\Theta-\Theta^{\prime}\right) d \Theta^{\prime}
$$



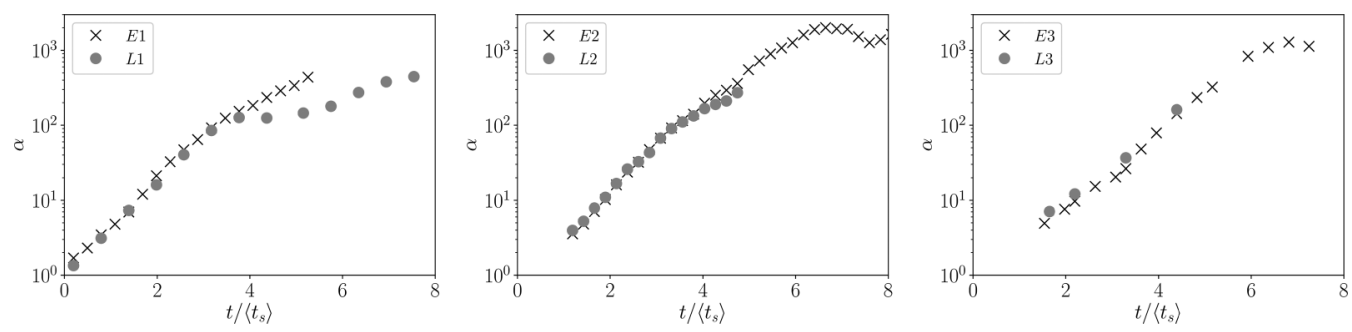

FIG. 10. Time dependence of the fit coefficient $\alpha$ for the scalar distribution exponential tails which have the shape $\exp (-\alpha \Theta)$ as shown in Fig. 6.

Equation (44) defines the convolution of the scalar PDF with itself. The resulting PDFs are added as gray lines in Fig. 9, where the number of self convolutions is indicated in the legend. A second self-convolution, $p^{\otimes 4}(\Theta, t)$, is understood as a subsequent self-convolution of $p^{\otimes 2}(\Theta, t)$ with itself, following the same rule Eq. (44) and so on. For the first time instant in Fig. 9(a), the predicted PDF as well as its first self-convolution overestimate the small concentrations as the comparison with the DNS data shows. This suggests that the filament formation out of the initial blob is still not mature enough to apply the model. For the second time instant in Fig. 9(b), the self-convolution overestimates again the low scalar concentration $\Theta$, but is in agreement with $p^{\otimes 2}(\Theta, t)$ with respect to high concentrations. In case of the third and fourth time instants in Figs. 9(c) and 9(d) of the same figure, the tails coincide partly with $p^{\otimes 4}(\Theta, t)$ and $p^{\otimes 6}(\Theta, t)$, respectively. Although this agreement is not perfect, we can state that the additional self-convolutions lead to an improved agreement of the tails of the predicted PDFs with those of the data. This is remarkable given the sequence of simplifications that have been made in the present model to obtain the PDF, such as the reduction to the maximum amplitude and the one-dimensionality of the model. To our knowledge these attempts of reconstructing the pdfs from stretching exponents and self-convolutions are the first of its kind for a three-dimensional turbulent field.

Finally, the time dependence of the exponential tails of numerical PDFs $P(\Theta, t)$ is analyzed. The tails are found to get sparser with increasing time. Also, the smaller Sc the faster the slope of the tails decreases with time. Similar to Ref. [20], we fit an exponential to the tail of the PDFs,

$$
P(\Theta, t) \propto \exp (-\alpha(t) \Theta) .
$$

For each data set, a least-square fit was done to obtain the parameter $\alpha\left(t /\left\langle t_{s}\right\rangle\right)$ as a function of normalized time $t /\left\langle t_{s}\right\rangle$. Figure 10 shows the dependence for all three cases in a logarithmic-linear plot. After a few mixing times $t /\left\langle t_{s}\right\rangle$ the fitting coefficients start to saturate at a constant value due to the overlap with sheets via the periodic boundaries which were described in the curves for scalar variance in Fig. 5.

\section{CONCLUSION AND OUTLOOK}

Direct numerical simulations were performed with a passive scalar concentration field mixed in a turbulent flow. The scalar field was represented in the Eulerian and the Lagrangian frames of reference, respectively. The Lagrangian case implies the advection of up to 4.8 billion individual tracer particles. One main goal of our study was a one-to-one comparison of the passive scalar mixing at Schmidt numbers Sc $\geqslant 1$ in these two frames of reference. Similar to a typical laboratory experiment, the passive scalar is initially injected in a small subvolume of the simulation domain. The central quantity of interest is the scalar concentration field $\Theta(x, t)$, which is either directly simulated as a field (Eulerian representation) or reconstructed from the tracer particle number in a each grid mesh cell (Lagrangian representation).

The comparison of both frames for the simplest case - a freely decaying passive scalar blob in a statistically stationary turbulent flow-confirms the applicability of the Lagrangian tracer particle model. A one-to-one comparison of the time evolution of the scalar variance and the scalar PDFs 
obtained in the whole simulation domain gives good agreement for $t \lesssim 10\left\langle t_{s}\right\rangle$ thus demonstrating the applicability of the Lagrangian model. The latter approach has advantages and disadvantages:

(i) For the Lagrangian runs at very large Schmidt number, it is in principle not necessary to increase the number of mesh points in the simulation run, since the Kolmogorov scale $\eta_{K}$ is well resolved. We used a higher resolution here to have a consistent one-to-one comparison with the corresponding Eulerian case. It is, however, necessary to evaluate the scalar concentration in the post-processing on a grid that matches the corresponding Batchelor scale $\eta_{B}$. This eases the computational effort in the Lagrangian case somewhat compared to the Eulerian one.

(ii) Effects of the noise due to discreteness and the variation of the number of tracer particles are absent in the Eulerian flow. Their impact grows in the Lagrangian runs as the tracer population is diluted with progressing time. This process prevents eventually a confirmation of the aggregation model at high Schmidt numbers (in particular dominant multi-layer aggregations as shown in Fig. 7) for times $t \gtrsim 10\left\langle t_{s}\right\rangle$ as done in laboratory experiments [19]. Such a study would require an ongoing adaptive local reseeding of tracer particles together with a removal of aged particles in a fully turbulent flow, i.e., a combination of the concepts that have been discussed in Refs. [23] and [29] for much simpler mixing cases in laminar flows.

Direct numerical simulations at high Schmidt numbers restrict the flow Reynolds number Re always to small or moderate values since a viscous-convective range has to be resolved. This is for example documented in the comprehensive data record in Ref. [46] for high-Schmidt-number mixing in homogeneous turbulence. It is shown there that the scalar variance increases with Sc at fixed Reynolds number. The dependence on Re is more complicated. The Reynolds number will affect the mixing time scale, higher Reynolds numbers will lead to shorter mixing times and higher strain rates. A further point is the mixing transition $[8,47]$. It states that the Reynolds number dependence of mixing processes is weaker once the turbulent flow has reached the regime of dissipative anomaly for which $\langle\epsilon\rangle L / u_{\mathrm{rms}}^{3}=$ const. The transition proceeds for $\operatorname{Re} \gtrsim 10^{4}$. Mixing experiments [48] support the existence of this transition. We expect, however, that the fundamental stretching and convolution dynamics of the sheets far below the Kolmogorov scale remains independent of this transition when rescaled in appropriate times and length units. A systematic study of this mixing transition by means of DNS at sufficiently high Sc is thus an important point for future work in this field.

The Lagrangian approach to passive scalar turbulent mixing made two further points possible: (1) Local stretching and compression rates (or positive and negative finite-time Lyapunov exponents) of the flow with their specific time history along fluid particle tracks can be calculated. A corresponding mean compression rate opens the definition of a mean mixing time $\left\langle t_{s}\right\rangle$-a typical time scale for the turbulent mixing process that was used to study their dependence on the Schmidt number. (2) A simplified one-dimensional local compression-diffusion process of a solitary strip allowed us to predict the scalar concentration PDF for shorter times and to obtain a behavior that agrees at least qualitatively with the data. The agreement was found to improve by including (multiple) selfconvolutions of the predicted PDF with itself which are based on the theory of random aggregation of scalar sheets [19]. In the future, such Lagrangian approach opens doors to more flexible boundary conditions. Other regimes, such as reseeding scenarios, periodic or steady point sources or several combined and localized sources, will thus be possible in future investigations.

\section{ACKNOWLEDGMENTS}

The work was supported by the Deutsche Forschungsgemeinschaft (DFG) in Germany and the Agence Nationale de la Recherche (ANR) in France with the joint grant TurbMix with Grants No. SCHU 1410/19-1 and No. ANR-14-CE35-0031-01, respectively. Supercomputing time has been provided at the JURECA supercomputer of the Jülich Supercomputing Centre (Germany) within the Project No. HIL10 of the John von Neumann-Institute for Computing. We thank Toshiyuki Gotoh, Patrice Meunier, and Jean-Luc Thiffeault for helpful discussions. 

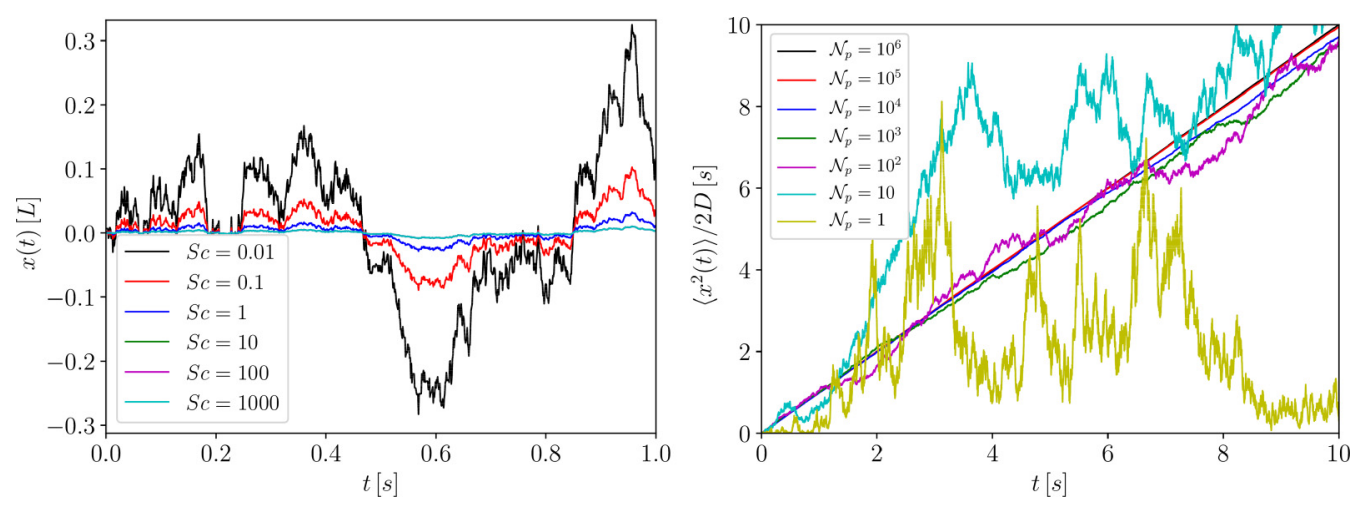

FIG. 11. Left: Test case for a stochastic Wiener process with different amplitudes $\sqrt{2 D}$ which correspond to different Schmidt number. Right: Verification of the Einstein relation for the mean square displacement with increasing number of realizations (or particles) for $\mathrm{Sc}=100$.

\section{APPENDIX: TEST AND IMPLEMENTATION OF THE STOCHASTIC INTEGRATION SCHEME}

Here, we report the test of the numerical implementation of the stochastic vectorial Wiener process $W_{j}(t)$ with $j=1,2,3$ that describes the diffusion in the Lagrangian framework [see Eq. (13)]. The Wiener process has two basic properties that have been already discussed in the main text. First, it has zero ensemble average. Second, it is white-in-time. The Langevin equation is given by [36]

$$
d X_{j}(t)=B_{j} d t+\sqrt{2 D} d W_{j}(t)
$$

with $B_{j}$ being the deterministic drift term. The numerical calculation with a time step width $\Delta t$ follows a second-order predictor-corrector scheme [49] and is given by

$$
\begin{gathered}
X_{j}^{*}=X_{j}^{n}+\Delta t B_{j}^{n}+\sqrt{2 D} \Delta W_{j}^{n}, \\
X_{j}^{n+1}=X_{j}^{n}+\frac{\Delta t}{2}\left[B_{j}^{n}+B_{j}^{*}\right]+\sqrt{\frac{D}{2}}\left[\Delta W_{j}^{n}+\Delta W_{j}^{*}\right],
\end{gathered}
$$

where $X_{j}^{n}$ is the tracer particle position after time $t_{n}=n \Delta t$. For each time step, each random number $\Delta W_{j}$ is calculated by

$$
\Delta W_{j}=\sqrt{\Delta t} Y_{j}\left(c_{1} Y_{j}^{2}+c_{2}\right)
$$

where $Y_{j}$ is a random variable generated between -0.5 and 0.5 for each space coordinate; $c_{1}=$ 14.14855378 and $c_{2}=1.21569221$. The amplitude of the random walk is thus controlled by the prefactor $\sqrt{2 D}$ as shown in the left panel of Fig. 11. The constants $c_{1}$ and $c_{2}$ are evaluated by solving a pair of quartic equations. More details on its solution can be found, for example, in Ref. [35] (see p. 137). For the test cases, we use different Schmidt numbers (or $D=v / S c$ with a value of $v$ from main text) to analyze the change of the amplitude of the random walk (with constant drift) of tracer particles. Furthermore, we test the number of tracer particles (or realizations of the Wiener process) that is necessary to accurately describe the Einstein relation for the mean square displacement for the case with a Schmidt number $\mathrm{Sc}=100$

$$
\left\langle X_{j}^{2}(t)\right\rangle=2 D t
$$

The results in the right panel of Fig. 11 show that a minimum of $10^{5}$ particles is required for a linear relation with respect to time. 
[1] A. M. Obukhov, Structure of the temperature field in a turbulent flow, Izv. Akad. Nauk SSSR, Ser. Geogr. Geofiz. 13, 58 (1949).

[2] S. Corrsin, On the spectrum of isotropic temperature fluctuations in an isotropic turbulence, J. Appl. Phys. 22, 469 (1951).

[3] G. K. Batchelor, Small-scale variation of convected quantities like temperature in turbulent fluid Part 1. General discussion and the case of small conductivity, J. Fluid Mech. 5, 113 (1959).

[4] R. H. Kraichnan, Small-scale structure of a scalar field convected by turbulence, Phys. Fluids 11, 945 (1968).

[5] Z. Warhaft, Passive scalars in turbulent flows, Annu. Rev. Fluid Mech. 32, 203 (2000).

[6] G. Falkovich, K. Gawędzki, and M. Vergassola, Particles and fields in fluid turbulence, Rev. Mod. Phys. 73, 913 (2001).

[7] Z. Warhaft, Turbulence in nature and in the laboratory, Proc. Natl. Acad. Sci. USA 99, 2481 (2002).

[8] P. E. Dimotakis, Turbulent mixing, Annu. Rev. Fluid Mech. 37, 329 (2005).

[9] T. Gotoh and P. K. Yeung, Passive scalar transport in turbulence: A computational perspective, in Ten Chapters in Turbulence, edited by P. A. Davidson, Y. Kaneda, and K. R. Sreenivasan (Cambridge University Press, Cambridge, 2013), p. 87.

[10] B. L. Sawford and J.-F. Pinton, A Lagrangian view of turbulent dispersion and mixing, in Ten Chapters in Turbulence, edited by P. A. Davidson, Y. Kaneda, and K. R. Sreenivasan (Cambridge University Press, Cambridge, 2013), p. 132.

[11] P. K. Yeung and K. R. Sreenivasan, Spectrum of passive scalars of high molecular diffusivity in turbulent mixing, J. Fluid Mech. 716, R14 (2013).

[12] P. K. Yeung, S. Xu, D. A. Donzis, and K. R. Sreenivasan, Simulations of three-dimensional turbulent mixing for Schmidt numbers of the order 1000, Flow Turb. Combust. 72, 333 (2004).

[13] D. A. Donzis, K. R. Sreenivasan, and P. K. Yeung, The Batchelor spectrum for mixing of passive scalars in isotropic turbulence, Flow Turb. Combust. 85, 549 (2010).

[14] J. Schumacher and K. R. Sreenivasan, Geometric Features of the Mixing of Passive Scalars at High Schmidt Numbers, Phys. Rev. Lett. 91, 174501 (2003).

[15] G. Brethouwer, J. C. R. Hunt, and F. T. M. Nieuwstadt, Micro-structure and Lagrangian statistics of the scalar field with a mean gradient in isotropic turbulence, J. Fluid Mech. 474, 193 (2003).

[16] J. Schumacher, K. R. Sreenivasan, and P. K. Yeung, Very fine structures in scalar mixing, J. Fluid Mech. 531, 113 (2005).

[17] J. Schumacher and K. R. Sreenivasan, Statistics and geometry of passive scalars in turbulence, Phys. Fluids 17, 125107 (2005).

[18] D. Kushnir, J. Schumacher, and A. Brandt, Geometry of Intensive Scalar Dissipation Events in Turbulence, Phys. Rev. Lett. 97, 124502 (2006).

[19] E. Villermaux and J. Duplat, Mixing as an Aggregation Process, Phys. Rev. Lett. 91, 184501 (2003).

[20] J. Duplat, A. Jouary, and E. Villermaux, Entanglement Rules for Random Mixtures, Phys. Rev. Lett. 105, 034504 (2010).

[21] J. Duplat, C. Innocenti, and E. Villermaux, A nonsequential turbulent mixing process, Phys. Fluids 22, 035104 (2010).

[22] T. Le Borgne, P. D. Huck, M. Dentz, and E. Villermaux, Scalar gradients in stirred mixtures and the deconstruction of random fields, J. Fluid Mech. 812, 578 (2017).

[23] D. Martínez-Ruiz, P. Meunier, B. Favier, L. Duchemin, and E. Villermaux, The diffusive sheet method for scalar mixing, J. Fluid Mech. 837, 230 (2018).

[24] E. Villermaux, Mixing versus stirring, Annu. Rev. Fluid Mech. 51, 245 (2019).

[25] T. M. Antonsen Jr., Z. Fan, E. Ott, and E. Garcia-Lopez, The role of chaotic orbits in the determination of power spectra of passive scalars, Phys. Fluids 8, 3094 (1996).

[26] E. Balkovsky and A. Fouxon, Universal long-time properties of Lagrangian statistics in the Batchelor regime and their application to the passive scalar problem, Phys. Rev. E 60, 4164 (1999).

[27] B. I. Shraiman and E. D. Siggia, Scalar turbulence, Nature 405, 639 (2000).

[28] P. H. Haynes and J. Vanneste, What controls the decay of passive scalars in smooth flows? Phys. Fluids 17, 097103 (2005). 
[29] T. Okabe, B. Eckhardt, J.-L. Thiffeault, and C. R. Doering, Mixing effectiveness depends on the sourcesink structure: Simulation results, J. Stat. Mech. (2008) P07018.

[30] J. Duplat and E. Villermaux, Mixing by random stirring in confined mixtures, J. Fluid Mech. 617, 51 (2008).

[31] D. Buaria, P. K. Yeung, and B. L. Sawford, A Lagrangian study of turbulent mixing: Forward and backward dispersion of molecular trajectories in isotropic turbulence, J. Fluid Mech. 799, 352 (2016).

[32] J. Schumacher, K. R. Sreenivasan, and V. Yakhot, Asymptotic exponents from low-Reynolds-number flows, New J. Phys. 9, 89 (2007).

[33] J. Schumacher and B. Eckhardt, Evolution of turbulent spots in a parallel shear flow, Phys. Rev. E 63, 046307 (2001).

[34] D. Pekurovsky, P3DFFT: A framework for parallel computations of Fourier transforms in three dimensions, SIAM J. Sci. Comput. 34, C192 (2012).

[35] C. H. Oettinger, Stochastic Processes in Polymeric Fluids Tools and Examples for Developing Simulation Algorithms (Springer, Berlin, 1996).

[36] C. W. Gardiner, Stochastic Methods: A Handbook for the Natural and Social Sciences (Springer, Berlin, 2009).

[37] A. Pikovsky and A. Politi, Lyapunov Exponents: A Tool to Explore Complex Dynamics (Cambridge University Press, Cambridge, 2016).

[38] M. R. Allshouse and T. Peacock, Lagrangian based methods for coherent structure detection, Chaos 25, 097617 (2015).

[39] G. Haller, Lagrangian coherent structures, Annu. Rev. Fluid Mech. 47, 137 (2015).

[40] R. Betchov, An inequality concerning the production of vorticity in isotropic turbulence, J. Fluid Mech. 1, 497 (1956).

[41] W. T. Ashurst, A. R. Kerstein, R. M. Kerr, and C. H. Gibson, Alignment of vorticity and scalar gradient with strain rate in simulated Navier-Stokes turbulence, Phys. Fluids 30, 2343 (1987).

[42] F. Városi, T. M. Antonsen Jr., and E. Ott, The spectrum of fractal dimensions of passively convected scalar gradients in chaotic fluid flows, Phys. Fluid. A 3, 1017 (1991).

[43] P. Meunier and E. Villermaux, The diffusive strip method for scalar mixing in two dimensions, J. Fluid Mech. 662, 134 (2010).

[44] D. Gottlieb and S. Orszag, Numerical Analysis of Spectral Methods (Society for Industrial and Applied Mathematics, Philadelphia, 1977).

[45] W. E. Ranz, Applications of a stretch model to mixing, diffusion, and reaction in laminar and turbulent flows, AIChE J. 25, 41 (1979).

[46] D. A. Donzis, K. R. Sreenivasan, and P. K. Yeung, Scalar dissipation rate and dissipative anomaly in isotropic turbulence, J. Fluid Mech. 532, 199 (2005).

[47] P. E. Dimotakis, The mixing transition in turbulent flows, J. Fluid Mech. 409, 69 (2000).

[48] P. E. Dimotakis, R. C. Miake-Lye, and D. A. Papantoniou, Structure and dynamics of round turbulent jets, Phys. Fluids 26, 3185 (1983).

[49] P. K. Yeung and S. B. Pope, An algorithm for tracking fluid particles in numerical simulations of homogeneous turbulence, J. Comp. Phys. 79, 373 (1988). 